\title{
A rare costal tumor complicating a solitary osteochondroma
}

\author{
Mona Mlika, Manel Yahia, Sadok Boudaya, Aida Ayadi-Kaddour, \\ Tarek Kilani, Faouzi El Mezni
}

\begin{abstract}
Introduction: Chondrosarcoma complicating osteochondromatous lesions is a microscopic diagnosis which treatment is based on surgical excision. These tumors need a close followup because the recovery can be ascertained only after 10 years of follow-up. Case Report: A 19-year-old male who presented respiratory symptoms and whose past medical history was remarkable for a previously noted cartilaginous exostosis in multiple long bones. Physical and radiologic investigations showed a mass attached to the first rib. The anterior arch of the rib was excised en-bloc with a layer of soft tissue. Histopathological examination concluded to a grade I chondrosarcoma arising from an osteochondroma. Conclusion: Secondary chondrosarcoma complicating a benign lesion such as osteochondroma has been rarely reported in the rib. We aim to highlight the diagnostic and therapeutic challenges of this rare tumor by describing a new case of secondary costal chondrosarcoma.
\end{abstract}

Keywords: Chondrosarcoma, Osteochondroma, Surgical resection

Mona Mlika', Manel Yahia1', Sadok Boudaya², Aida AyadiKaddour ${ }^{1}$, Tarek Kilani ${ }^{2}$, Faouzi El Mezni ${ }^{1}$

Affiliations: ${ }^{1}$ Department of Pathology, Abderrahman Mami Hospital, University of Medicine, Tunis El Manar; ${ }^{2}$ Department of Thoracic Surgery, Abderrahman Mami Hospital, University of Medicine, Tunis El Manar.

Corresponding Author: Mona Mlika, Deparment of Pathology. Abderrahman Mami Hospital; Ph: (00216) 985388 62; Email: mlika.zorgati.mona@hotmail.com

Received: 22 December 2012

Accepted: 18 February 2013

Published: 01 June 2014

\section{How to cite this article}

Mlika M, Yahia M, Boudaya S, Ayadi-Kaddour A, Kilani T, El Mezni F. A rare costal tumor complicating a solitary osteochondroma. Int J Case Rep Images 2014;5(6):413-416.

doi:10.5348/ijcri-201478-CR-10389

\section{INTRODUCTION}

Secondary chondrosarcoma is a tumor arising in a benign precursor lesion, either an osteochondroma or an enchondroma [1]. The risk of chondrosarcoma in a solitary osteochondroma has been reported to be $2 \%$, and $5-25 \%$ in osteochondromatosis [2-4]. Secondary chondrosarcoma have a predilection for flat bones and is more frequently observed in the third decade of life, compared with chondrosarcoma arising de novo in which the peak incidence is within the fourth to the sixth decade [5]. We present a young patient with multiple exostoses who had a rare location of secondary chondrosarcoma arising on the first right rib and we discuss the causal relationship between the two lesions on the basis of the radiological and pathological findings.

\section{CASE REPORT}

A 19-year-old male patient presented four months ago with intermittent and progressively increasing chest pain associated to a fever and multiple subcutaneous nodes. The past medical history of our patient was remarkable for a previously noted cartilaginous exostosis in multiple long bones. His family history revealed multiple cervical bony lesions in her sister; who had been operated 4 years ago. Physical examination revealed multiple bony swelling at the metaphysic ends of all long bones. A large $(4 \mathrm{~cm})$ firm and slightly tender mass was palpated in the right infraclavicular area. The mass seemed to be 
attached to the first rib without extension to the overlying skin. Chest X-ray showed a bony and heterogeneous lesion developed in the first right rib, irregularly shaped and measuring $4 \mathrm{~cm}$ (Figure $1 \mathrm{~A}$ ).

Computed tomography (CT) scan showed a degenerated exostosis of the first rib associated with multiple axillary lymph nodes and subcutaneous nodes (Figure 1B). This process was highly suggestive of a malignant process. Subsequently, the anterior arc of the rib was excised en-bloc with a layer of soft tissue. Grossly, the rib measured $7 \times 2 \times 1.5 \mathrm{~cm}$ and was centered by a tumor which had a hard consistency (Figure 1C). Microscopic examination showed a malignant and well differentiated cartilage proliferation consisting in irregularly shaped lobules of cartilage separated by fibrous bands (Figure 1D). This proliferation permeates bony trabeculae, cartilage cap and surrounding soft tissues. Within the tumor, the cellularity was mildly increased. The chondrocytes showed varied size and shape (Figure $1 \mathrm{E}-\mathrm{F}$ ). No mitotic figures were present. Extensive calcifications were seen. Some specimen showed lesions of osteochondroma

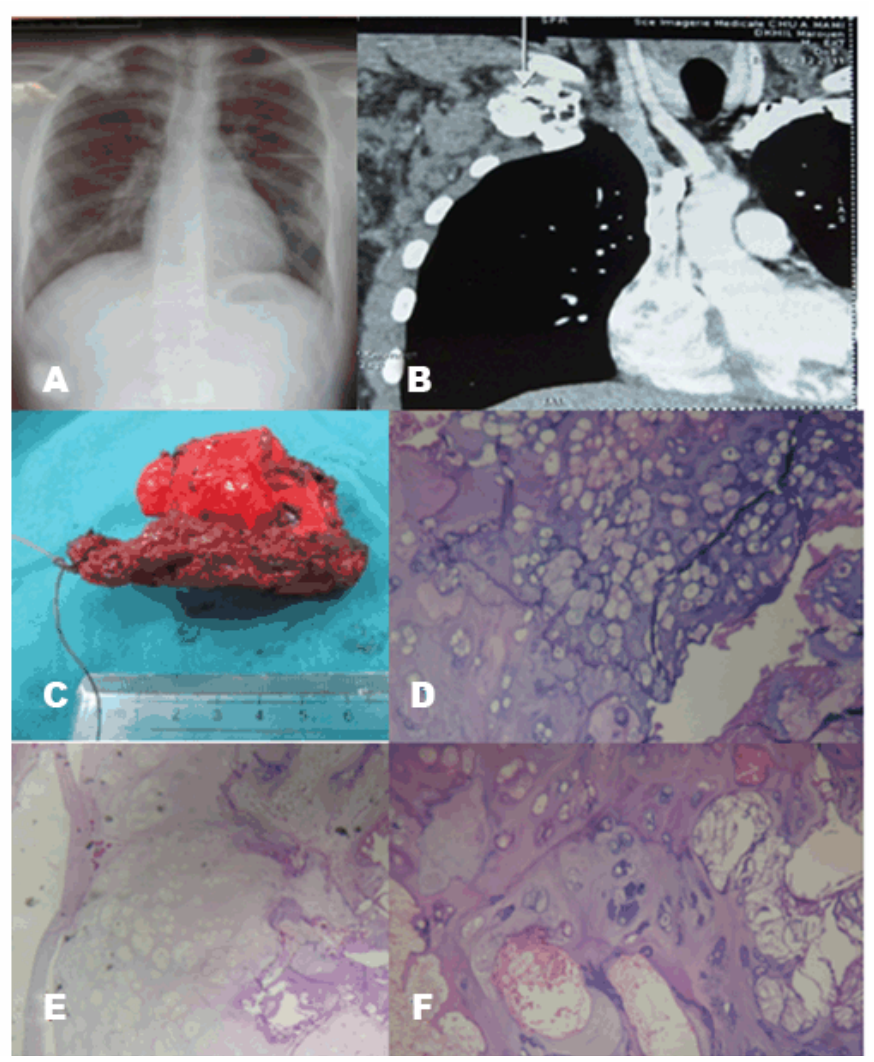

Figure 1: (A) Chest-X-ray showing a heterogeneous and irregularly shaped lesion developed in the first right rib (arrow), (B) Computed tomography scan of chest showing a costal lesion extending to the adjacent structures (arrow), (C) Gross appearance of a costal lesion measuring $4 \mathrm{~cm}$ invading the rib, (D) Microscopic findings showing a malignant proliferation characterized by irregularly shaped lobules of cartilage separated by fibrous bands (H\&E stain, x25O). (E) Microscopic findings showing the permeation of the cartilage cap by the malignant proliferation (H\&E stain, x25O). (F) Microscopic findings showing polymorphous chondrocytes with increased cellularity (H\&E stain, x400). consisting from the outside to the inside in fibrous bands, cartilage cap with cells without atypical figures and a bony trabeculae tissue. The final diagnosis retained was a grade I chondrosarcoma arising from an osteochondroma.

\section{DISCUSSION}

Osteochondromas are the most common benign bone tumors that occur in approximately $3 \%$ of the population with predominance in young male adults $[1,2]$. Patients usually remain asymptomatic $[2,5,6]$. These tumors are often solitary cartilaginous exostoses located in the distal femur, the proximal tibia or fibula, or the proximal humerus $[2,7]$. They may also arise as multiple lesions within the scope of the hereditary multiple exostoses disorder or gene-deletion syndromes [2]. Most lesions stagnate and ossify when skeletal growth is complete, but occasionally, they might grow more aggressively into a low-grade chondrosarcoma [2, 7]. Almost one-third of all chondrosarcomas arise secondarily [2]. Uncommon before 20 years, secondary chondrosarcoma arise mainly after 40 years $[2,5]$. Any portion of the skeleton may be involved. However, the pelvis and shoulder girdle bones are more frequently affected $[1,3,8,9]$. Costal localization, like our case, is an unusual site for this tumor. Less than 20 costal chondrosarcoma secondary to osteochondroma have been reported in English literature. Secondary chondrosarcoma is suspected by growth of the tumor after puberty, the presence of pain, or a thickness over $1 \mathrm{~cm}$ of the cartilaginous cap in adults $[4,5]$. In our case, the patient noticed a growing painful mass. The CT scan is helpful for delineating the thickness of the cartilage cap and the search of a cortical destruction [10]. In our case, chest X-ray and CT scan showed a suspected lesion at the bottom of a cartilaginous exostosis with irregular cartilage cap. Although the importance of clinical and radiologic data, the diagnosis is based on histopathological findings. Means of diagnosis for microscopic analysis are still debated. Some authors suggest per cutaneous biopsy, but others prefer, a large resection of the tumor. The latter approach allows a better analysis of the entire lesion with the resection margins and minimizes the risk of metastasis and recurrences [5]. Grossly, chondrosarcoma secondary to osteochondroma are characterized by a thickness superior to $2 \mathrm{~cm}$. These lesions are generally lobulated and usually show cystic cavities. Secondary chondrosarcoma is usually very myxoid and hence appears mucoid. This contrasts with the solid blue matrix of the areas of chondromatosis. Microscopically, secondary chondrosarcoma is frequently low-grade tumor with few regular cells. Invasion of the surrounding tissues and marked myxoid change in the matrix are helpful diagnostic features [1]. The treatment of secondary chondrosarcoma is based on a wide surgical excision [3, 5]. Adequate removal of the tumor including the upon and underlying intercostal spaces with resection margin of $4 \mathrm{~cm}$ is required for treatment and prevention from 
local recurrence [5]. In our case the resection margins were tumoral, that's why, and a resection of the second rib is planned. Adjuvant chemotherapy and radiotherapy are mainly used for the treatment of inoperable patient or in case of recurrence $[3,5]$. The quality of resection is the most relevant prognostic factor $[3,5]$. However, other prognostic factors are evocated such as the grade of the tumor, its site and the lesion cutting [5]. Recent estimates suggest a lifetime risk of about $2-4 \%[7,9]$. A prolonged survey is necessary because the recovery of chondrosarcoma cannot be asserted before 10 years of follow-up.

\section{CONCLUSION}

Secondary chondrosarcoma complicating a benign lesion such as osteochondroma has been rarely reported in the rib. We aim to highlight the diagnostic and therapeutic challenges of this tumor. By describing a new case of secondary costal chondrosarcoma. In fact, the diagnosis is based on histopathological examination but clinical and radiologic findings are helpful in showing the malignant nature of the tumor. Although based mainly on surgical treatment, the management of these tumors remains difficult necessitating a narrow follow-up because the recovery can be ascertained only after 10 years.

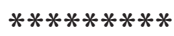

\section{Acknowledgements}

Written consent was obtained from the patients for permission for the publication of this case report. Thank you for your attention to our paper.

\section{Author Contributions}

Mona Mlika - Substantial contributions to conception and design, Acquisition of data, Analysis and interpretation of data, Drafting the article, Revising it critically for important intellectual content, Final approval of the version to be published

Manel Yahia - Analysis and interpretation of data, Revising it critically for important intellectual content, Final approval of the version to be published

Sadok Boudaya - Analysis and interpretation of data, Revising it critically for important intellectual content, Final approval of the version to be published

Aida Ayadi-Kaddour - Analysis and interpretation of data, Revising it critically for important intellectual content, Final approval of the version to be published Tarek Kilani - Analysis and interpretation of data, Revising it critically for important intellectual content, Final approval of the version to be published

Faouzi El Mezni - Analysis and interpretation of data, Revising it critically for important intellectual content, Final approval of the version to be published

\section{Guarantor}

The corresponding author is the guarantor of submission.

\section{Conflict of Interest}

Authors declare no conflict of interest.

\section{Copyright}

(C) 2014 Mona Mlika et al. This article is distributed under the terms of Creative Commons Attribution License which permits unrestricted use, distribution and reproduction in any medium provided the original author(s) and original publisher are properly credited. Please see the copyright policy on the journal website for more information.

\section{REFERENCES}

1. Bertoni F, Bacchini P, Hogendoom PCW. Chondrosarcoma. In: Fletcher CDM, Uni KK, Mertens F. Pathology and Genetics of Tumors of Soft Tissue and Bone: World Health Organization Classification of Tumors. Lyon, France: IARC Press; 2002.p.14654 .

2. Meissner SA, Vieth V, August C, Winkelmann W. Radiology-pathology conference: osteosarcoma in a cartilaginous exostosis of the femur. Clin Imaging 2006;30(3):206-9.

3. Chadha M, Singh AP. Secondary chondrosarcoma of the cuboid bone in a patient with multiple exostoses. Can J Surg 2008;51(1): E5-6.

4. Bovée JV, Sakkers RJ, Geirnaerdt MJ, Taminiau AH, Hogendoorn PC. Intermediate grade osteosarcoma and chondroma. A case report of a patient with hereditary multiple exostoses. J Clin Pathol 2002;55(3):226-9.

5. Morin B, Le Rochais JP, Galateau F, Icard P. [Costal chondroma and chondrosarcoma]. Ann Chir 2000;125(8):773-5.

6. Hudson TM, Chew FS, Manaster BJ. Scintigraphy of benign exostoses and exostotic chondrosarcomas. AJR Am J Roentgenol 1983;140(3):581-6.

7. Porter DE, Lonie L, Fraser M, et al. Severity of disease and risk of malignant change in hereditary multiple exostoses. A genotype-phenotype study. J Bone Joint Surg Br 2004;86(7):1041-6.

8. Wicks IP, Fleming A. Chondrosarcoma of the calcaneum and massive soft tissue calcification in a patient with hereditary and acquired connective tissue diseases. Ann Rheum Dis 1987;46(4):346-8.

9. Heffernan EJ, Alkubaidan FO, Munk PL. Radiology for the surgeon: musculoskeletal case 42. Chondrosarcoma arising from an osteochondroma in a patient with hereditary multiple exostoses (osteochondromatosis). Can J Surg 2008;51(5):3978.

10. Bernard SA, Murphy MD, Flemming DJ, Kransdorf MJ. Improved differentiation of benign osteochondromas from secondary chondrosarcomas with standardized measurement of cartilage cap at CT and MR imaging. Radiology 2010;255(3):857-65. 
Access full text article on other devices

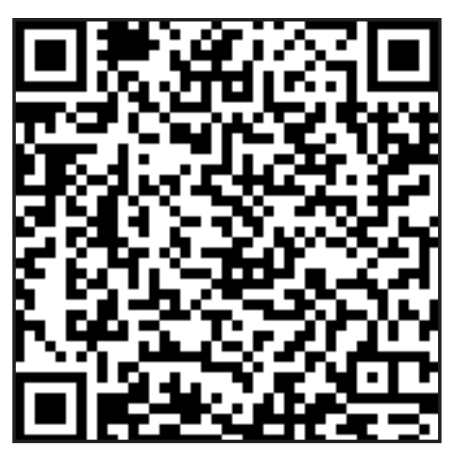

Access PDF of article on other devices

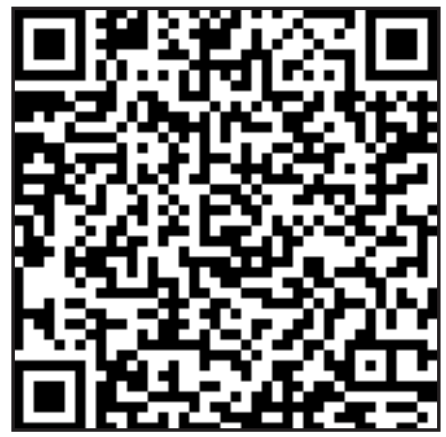

